\title{
Analyze and Compare the Function of Several Common Operators in Feature Extraction on Cotton Diseases
}

\author{
Jianchi Sun ${ }^{1, a}$ and Cao Yuan ${ }^{1, b^{*}}$ \\ ${ }^{1}$ School of Mathematic \& Computer Science. Wuhan Polytechnic University, Wuhan China \\ aJianchi Sun@gmail.com, byuancao1980@gmail.com \\ ${ }^{*}$ The corresponding author
}

Keyword: Feature extraction; Cotton leaf; Analyze and compare; Operator

\begin{abstract}
Cotton is the main plant of braided fabric in China, the production of cotton planting is important, but cotton are caused by diseases and insect pests easily in planting, so in the process of farming, some useful information of cotton diseases should be achieved easily and can be analyzed effectively, so some accurate and effective actions can be done. In the paper, cotton leaf as the research object, the main diseases are introduced, how to detect the main diseases is the important step in modern agricultural digital technology. In the paper, cotton leaf as the object of research, the main features of apple leafs should be reserved, and the special features of the disease region in apple leaf should be emphasized effective. The novel algorithm based on color components and edge extraction algorithms. More than 500 results of main cotton image with the main diseases can be compared by main operator algorithms and color components, analyze the results of different cotton diseases and operators, the new algorithm based on color difference and morphological features be precedence than other algorithms in the main cotton diseases.
\end{abstract}

\section{Introduction}

With the development of modern digital technology, cotton as the main plant of braided fabric in China, the production of cotton planting is important, but cotton are caused by diseases and insect pests easily in planting, so in the process of farming, some useful information of cotton diseases should be achieved easily and can be analyzed effectively, so some accurate and effective actions can be done.

How to detect the cotton diseases effectively is the important step in modern agricultural digital technology. The key of the detection technology is the feature extraction on cotton diseases.

In the paper, the main cotton diseases were introduced in detail, and four common operators Sobel, Roberts, Prewitt and Canny were used to detect the edge of leaves of different cotton diseases, which based on color components for the detection of cotton diseases.

Analyze and compare more than 500 results based on the various operators on the different cotton diseases. Some effective ways and rules can be deduced, that can improve the affection of detection in $\backslash$ the cotton diseases.

\section{The Main Diseases of Cotton Leaf}

There are three diseases of cotton leaf in the planting, the cotton fusarium and verticillium wilts, image of cotton leaf spot and cotton wheel spot disease. The normal image of cotton image and these three main diseases images of cotton leaf are shown in the figures from the image 1 to image 4.

In the paper, the main four operators of image is used to detect the image of cotton image, the Sobel, Roberts, Prewitt and Canny algorithms, are the most important in image edge detection operators in machine learning, digital media, computer vision, and other areas of the information technology plays an important role. 
Technically, it is a discrete first-order difference operator, a ladder is used to calculate the image brightness function degree of approximation. In any of the image using this operator, will produce a corresponding gradient vector or the point at which a method of vector.

\section{Methods}

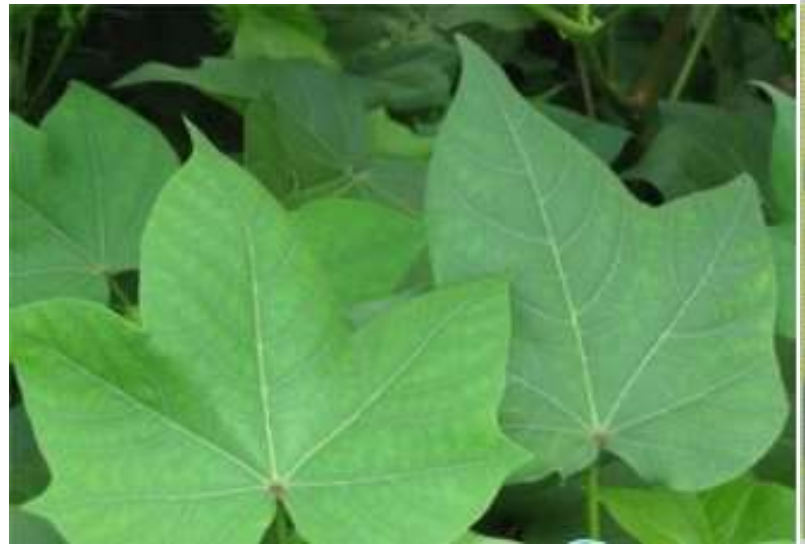

Figure 1. the normal image of cotton image

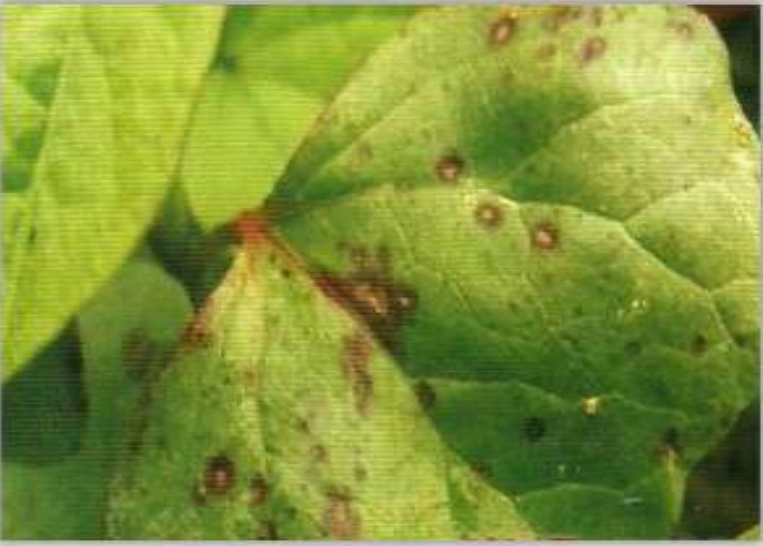

Figure 2. the image of cotton fusarium and verticilliumwilts
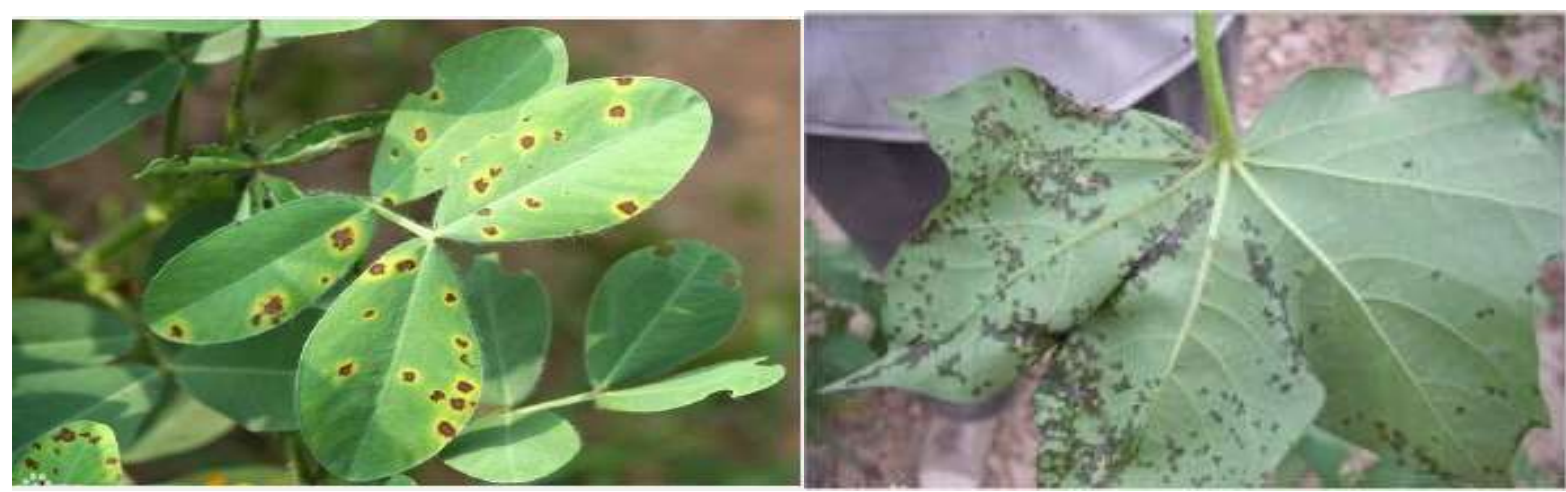

Figure 3. the image of cotton leaf spot

Figure 4. the image of cotton wheel spot disease.

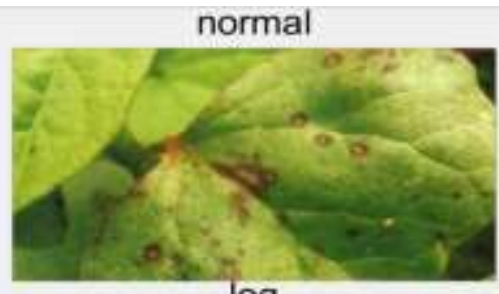

$\log$

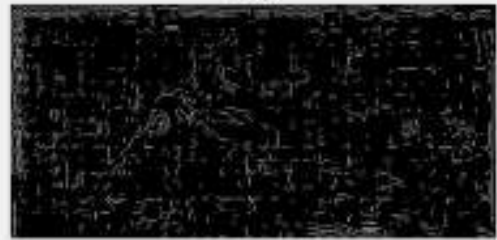

canny

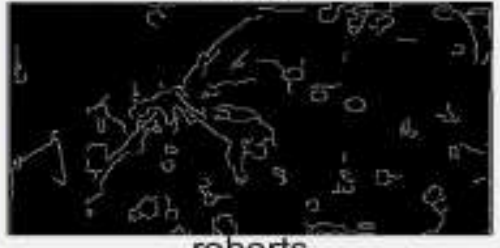

roberts

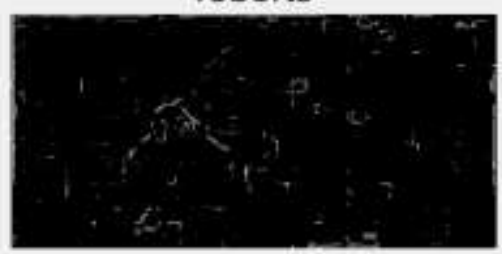

sobel

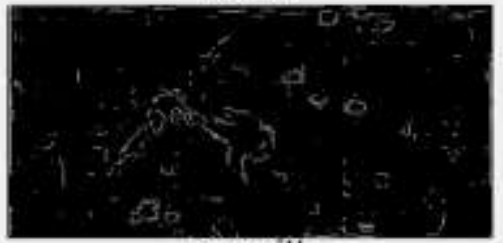

prewitt

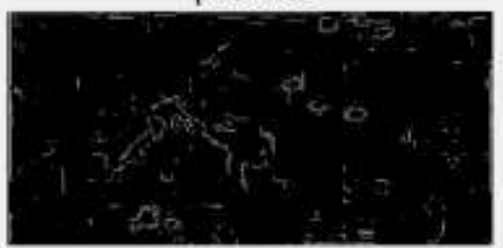

Figure 5. the image of cotton angular leaf spot

Figure 6. The result image of cotton fusarium and verticillium wilts disease based on Sobel,Log, Roberts, Prewitt and Canny operator. 

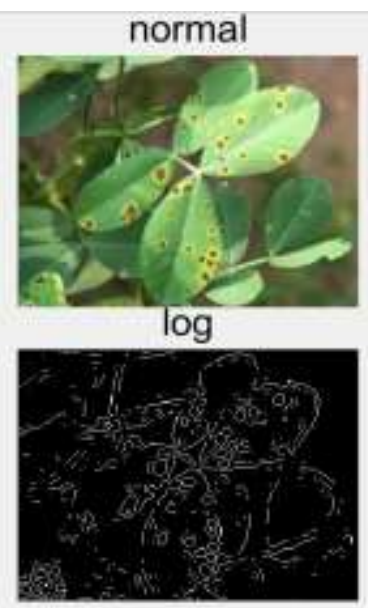

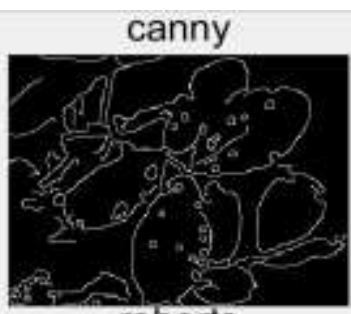

roberts

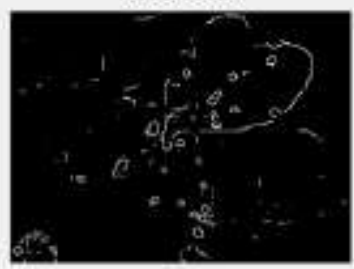

sobel

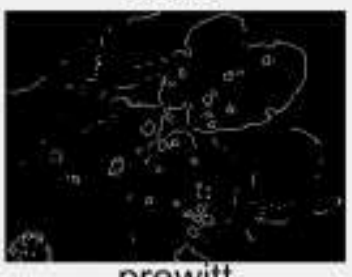

prewitt

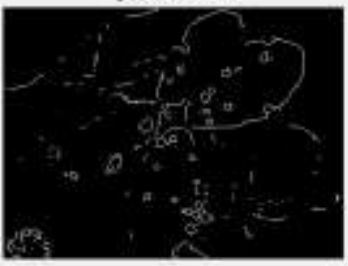

Figure 7. The result image of cotton leaf spot disease based on Sobel, Log, Roberts, Prewitt and Canny operator/
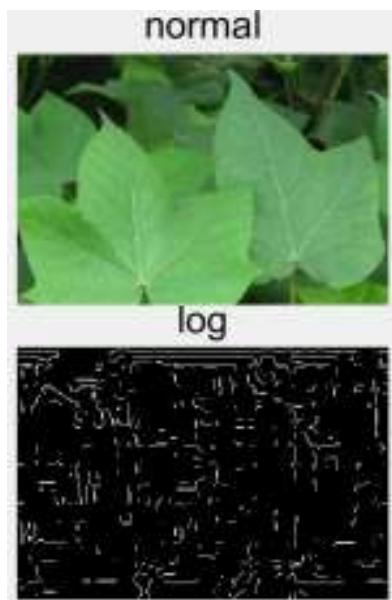

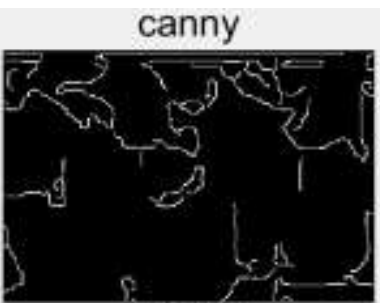

roberts

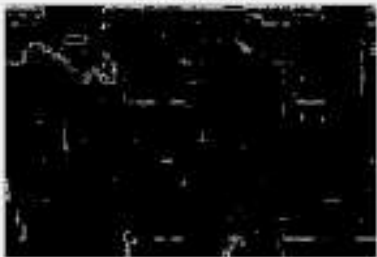

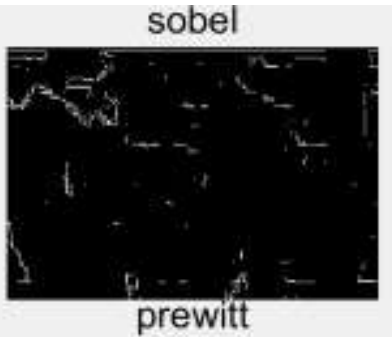

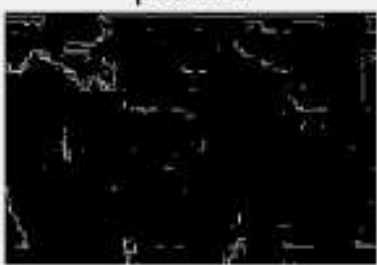

Figure 8. The result image of normal cotton leaf based on Sobel, Log, Roberts, Prewitt and Canny operator.

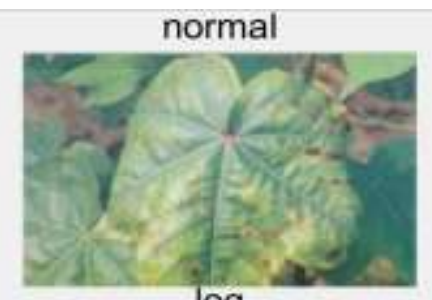

$\log$

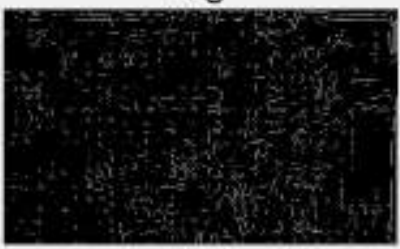

canny

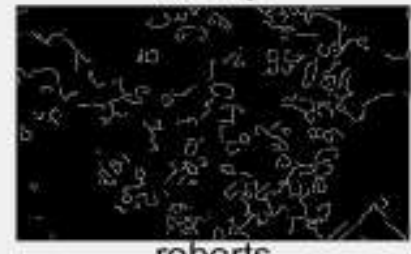

roberts

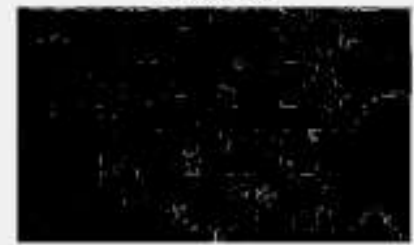

sobel

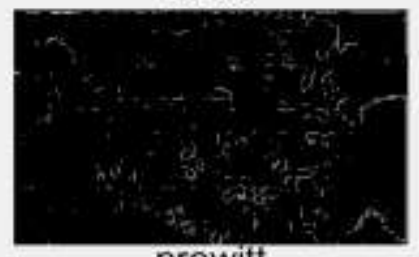

prewitt

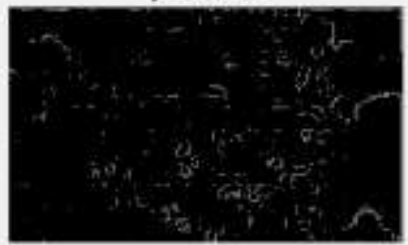

Figure 9. The result image of cotton wheel spot disease based on Sobel, Log, Roberts, Prewitt and Canny operator 


\section{Results}

The main in cotton planting, the Canny operator is better used in feature extraction on cotton leaf diseases. The extract edge can not be detected easily, but the leaf edge of disease region in cotton leaf is can be emphasized easily, so the novel algorithm should be combined with the edge of the color separation after edge extraction algorithm is more efficient

Analyze more than 500 results, the disease region of image can be more prominent based on color separation algorithm, the accurate of green cotton leaf is better than other color cotton leafs. So the algorithm be suit for detect the green cotton leaf base on green color separation algorithm.

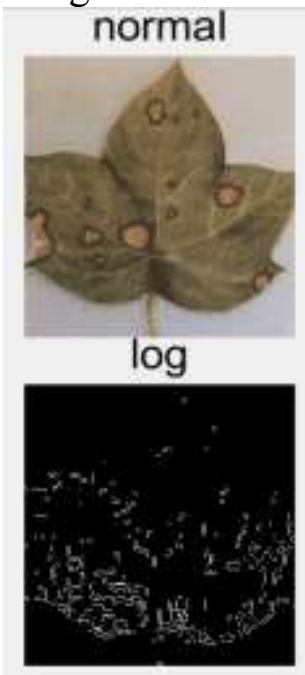

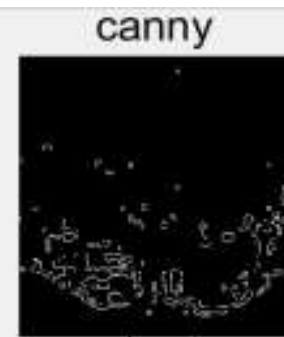

roberts

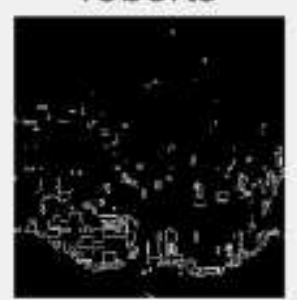

sobel

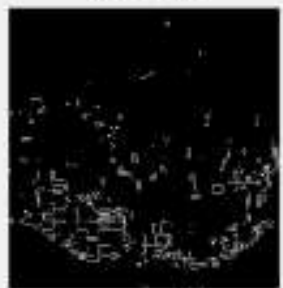

prewitt

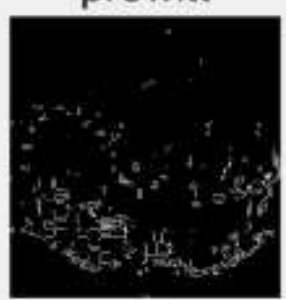

Figure 10. The result image of green cotton based on Sobel, Log, Roberts, Prewitt and Canny operators with combine of on green color separation algorithm.

\section{Acknowledgement}

This work was supported by the Natural Science Foundation of Hubei Province of China under Grant No.2016CFB470.

\section{References}

[1] Chandler D M,Hemami S S.VSNR:a wavelet-based visual signal-to-noise ratio for natural images[J].IEEE Transactions on Image Process,2007,16(9):2284-2298.

[2] Wang Z,Bovik A C,Sheikh H R,et a1.Image quality assessment:From error visibility to structural similarity[J].IEEE Transactions on Image Process,2004,13(4):600-612

[3] Zhang L,Zhang L,Mou X Q,et a.FSIM:a feature similarity index for image quality assessment[J].IEEE Transactions on Image Processing,2011,20(8):2378-2386.

[4] Jain R,Kasturi R,Schunck B G.Machine vision[M].[s.I.]:McGraw-Hill,Inc.1995.

[5] Jahne B,Haubecke H R,Geible P R.Handbook of computer vision and applications[M]. [s.1.]:Academic Press, 1999.

[6] Vrindts E, Reyniers M, Darius P,et.a1.Analysis of soil and crop properties for precision agriculture for winter wheat[J].Biosystems Engineering,2003,85(2):141-152

[7] Zhang Z Y.Flexilbe camera calibration by viewing a plane from unknown orientation[C]//Proc of ICCV99.[s.1.]:[s.n.],1999:51-52. 